\title{
Comparison of intralobar non-parenchyma, subcapsular non-parenchyma, and liver capsule thickness
}

\author{
J W RYOO, R J BUSCHMANN \\ From the Departments of Pathology, University of Illinois at Chicago, and Veterans Administration West Side \\ Medical Center, Chicago, Illinois, United States of America
}

SUMMARY The intralobar and subcapsular areas of human liver were compared in terms of morphometry and morphology, and a correlation was made between the thickness of liver capsules and the amount of intralobar non-parenchyma in normal and diseased livers. Tissue was systematically sampled from 16 normal and 26 fibrotic livers taken at necropsy. The volume fraction of subcapsular and intralobar non-parenchyma and the capsule thickness were determined by morphometry on histological sections. The volume fraction of intralobar non-parenchyma not only correlated well $(\tau=0.7, \mathrm{p}<0.001)$ with but was also equivalent in each case to the actual volume fraction of subcapsular non-parenchyma. Morphologically, the intralobar and subcapsular areas were remarkably similar. The volume fraction of intralobar non-parenchyma also correlated well $(\tau$ $=0.5, \mathrm{p}<0.001$ ) with capsule thickness.

These results show that pathological changes in liver architecture were essentially similar in the intralobar and subcapsular areas and that capsule thickness reflected intralobar non-parenchymal changes.

Previous morphometric analyses of fibrosis in rat livers have shown that not only is total liver capsule volume remarkably and significantly increased from $0 \cdot 27 \%$ to $1 \cdot 4 \%$ of total liver volume in cirrhosis ${ }^{1}$ but that there is good correlation (correlation coefficient $=0.87$ ) between histologically measured capsule thickness and the degree of fibrosis. ${ }^{2}$ Although capsule thickening in liver disease is known to occur, the correlation of capsule thickness with overall liver structure has not been studied. The continuity and ramification of the capsular stroma into the portal tracts and into the connective tissue of the hepatic veins $^{3}$ makes such a correlation reasonable, but many pathologists are concerned about the use of a subcapsular sample for histological assessment of liver changes because non-parenchyma may be overly represented compared with the rest of the liver. ${ }^{46}$ Although the concern is often mollified, ${ }^{6-8}$ there is a need for a quantitative comparison of the amount of non-parenchyma in the intralobar and subcapsular areas. We report here the results of a study in which we made this comparison as well as measuring capsule thickness in human liver.

Accepted for publication 16 March 1989

\section{Material and methods}

Liver tissue was sampled during consecutive necropsies at two medical centres except for cases with gross liver disease with neoplasia. A total of $\mathbf{4 2}$ livers was sampled from 35 men and seven women. The average (SD) age was $62(15)$ years and average liver weight was $1600(600) \mathrm{g}$. There were 16 normal and 26 fibrotic (14 cirrhotic) livers.

The right lobe was sliced parallel to the visceral surface (usually two slices) and fixed in a buffered $4 \%$ formaldehyde solution. The right lobe was chosen because it is the lobe that is usually sampled by needle biopsy. Three pieces of tissue measuring $2 \times 5 \times 10$ $\mathrm{mm}$ deep (from and perpendicular to the liver surface) were taken from each slice. The pieces were sampled at roughly $120^{\circ}$ intervals around each cut surface, with the sampling at one slice offset $60^{\circ}$ from the other slice. Areas of the coronary and falciform ligaments as well as adhesions were avoided. Three corresponding and similarly sized pieces of intralobar tissue (at least $2 \mathrm{~cm}$ away from the capsule or hilar area) were taken from a cut surface of each slice. The pieces of tissue were embedded in paraffin wax, sectioned perpendicular to the liver surface, and stained with a "liver trichome stain" (HISTO-LOGIC) ${ }^{9}$ for morphological and morphometric analysis. 
The volume fractions $\left(V_{v}\right)$ of subcapsular and intralobar non-parenchyma for each liver were estimated for each case by point counting. ${ }^{10} \mathrm{~A}$ light microscope (at a magnification of 63 times) and with a camera lucida attachment was used to project a $4 \times 4$ square lattice grid on to the field of view, which covered a $3 \mathrm{~mm}$ diameter area of tissue. The 16 grid intersections were spaced at $0.4 \mathrm{~mm}$ intervals and were used to count points on parenchyma and nonparenchyma (segmental blood vessels and bile duct lumens with diameters larger than one third of the lattice spacing were not counted). For the subcapsular area, three consecutive fields, which started immediately beneath the capsule and extended perpendicularly to a depth of about $1 \mathrm{~cm}$, were measured in a section from each of three randomly chosen blocks. For the intralobar area, three consecutive fields starting conventionally with the left lower corner of the section were similarly measured for each of three randomly chosen blocks.

Capsule thickness was defined as the perpendicular distance between the liver surface and lobular or nodular parenchyma. Areas of local extra-capsular fibrosis like adhesions were not included in the measurements. In the case of nodular parenchyma the distance was measured from the liver surface to a line drawn more or less tangential to and successively from the outermost aspects of each nodule in the particular section. A light microscope (at a magnification of 63 times) with a camera lucida attachment over an $x-y$ digitising tablet was used to project an image of four parallel lines from a drawing placed on the tablet on to the field of view in the microscope (line spacing at 0.4 $\mathrm{mm}$ ). The lines were randomly oriented perpendicular to the capsular surface; capsule thickness was measured along each line on the digitising tablet with a

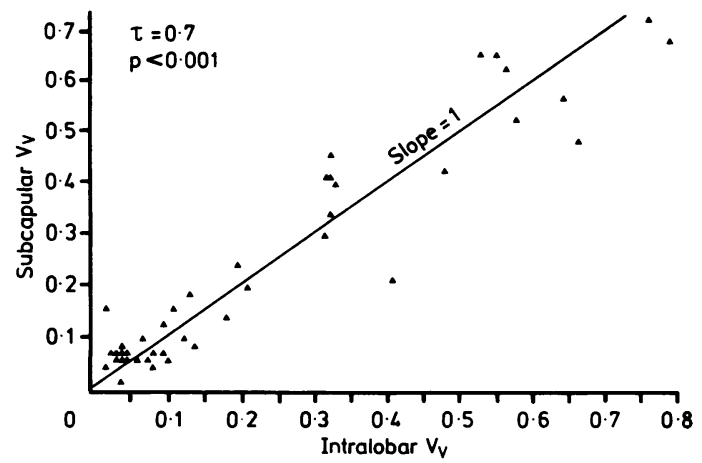

Fig 1 Scatter plot of volume fractions $\left(V_{V}\right)$ of subcapsular versus intralobar non-parenchyma for each of the 42 cases studied. The line indicates a slope of 1 , which represents a one-to-one correspondence between the intralobar and subcapsular values. light-emitting diode cursor connected to a microcomputer (BioQuant II, R\&M Biometrics, Nashville, Tenessee, USA). Sections from each of three randomly chosen blocks were measured for each case.

The Non-parametric Statistics Program of BMDP Statistical Software (Los Angeles, California, USA) was used to find the Kendall's coefficient of rank correlation between variables.

\section{Results}

The volume fraction of intralobar non-parenchyma correlated well $(\tau=0.7, p<0.001)$ with the volume fraction of subcapsular non-parenchyma. Moreover, there was little, if any, difference in the actual volume fraction between the subcapsular and intralobar areas in each case; this equality was apparent from the distribution of the data points for each case around a line with a slope (1) (fig 1).

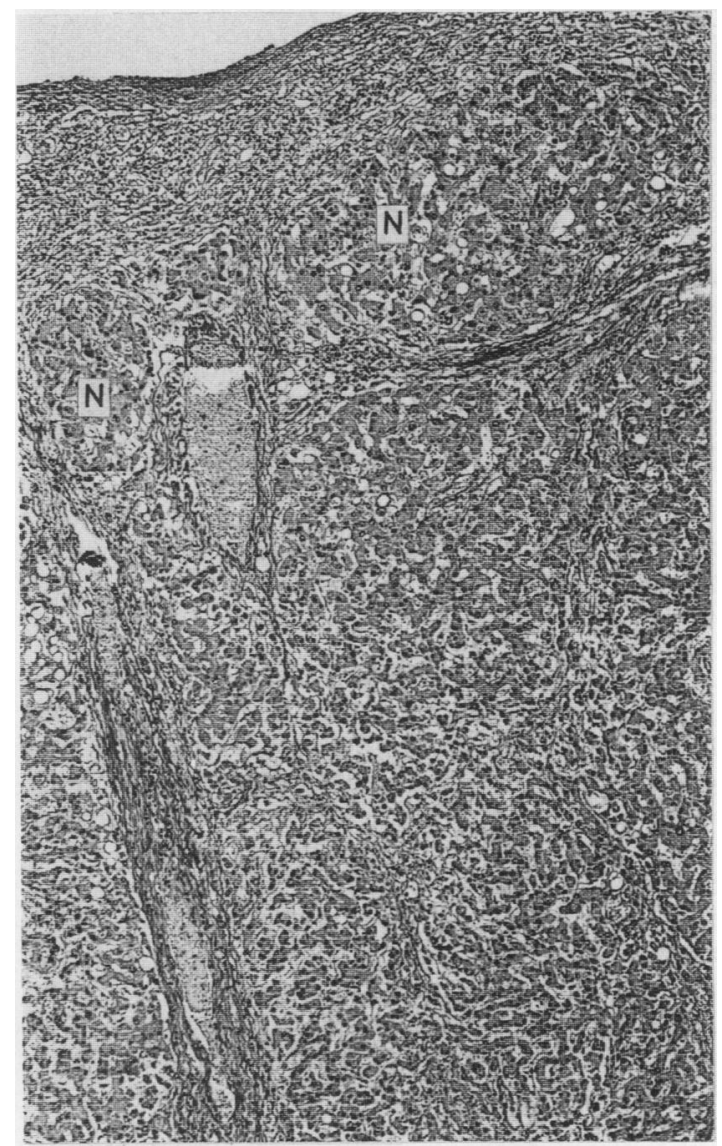

Fig 2 Subcapsular area of a non-cirrhotic liver shows apparent nodules $(N)$ immediately beneath the liver capsule. Most of the subcapsular area, however, is without nodules. ("Liver trichrome stain".) 


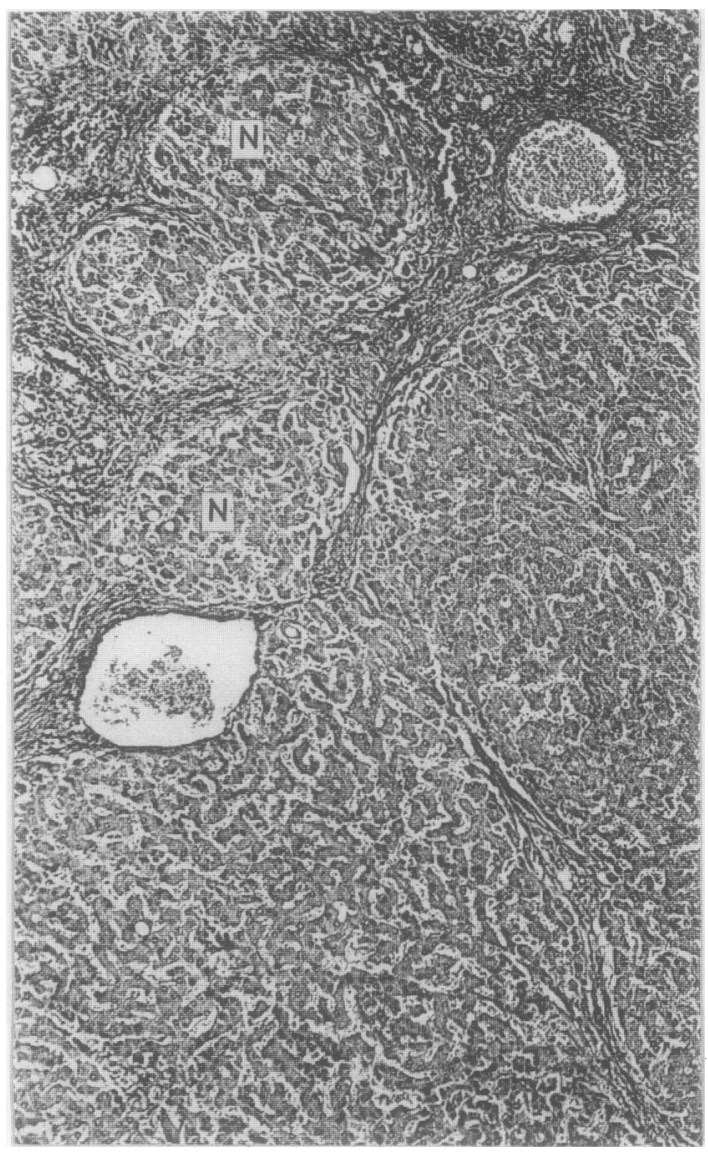

Fig 3 Intralobar area of a non-cirrhotic liver shows apparent nodules $(N)$ that were found only at sites immediately adjacent to large portal tracts. ("Liver trichrome stain".)

In accord with the morphometric data, histological examination showed no apparent differences in the amount of fibrosis and in the prevalence of nodules between intralobar and subcapsular areas. The only potential source of error in cases of fibrosis without cirrhosis was the occasional small area of parenchyma that was immediately beneath the capsule and walled off by non-parenchymatous tracts (fig 2), and which gave the impression of nodulation. This resembled intralobar nodules adjacent to major portal tracts (fig 3 ). In these cases the greater part of the intralobar and subcapsular samples were clearly without nodules, and consequently the livers were evidently not cirrhotic.

Capsule thickness was also correlated well $(\tau=0.5$, $p<0.001$ ) with the volume fraction of intralobar nonparenchyma (fig 4). Morphological details of the capsules tended to show that the major thickening was

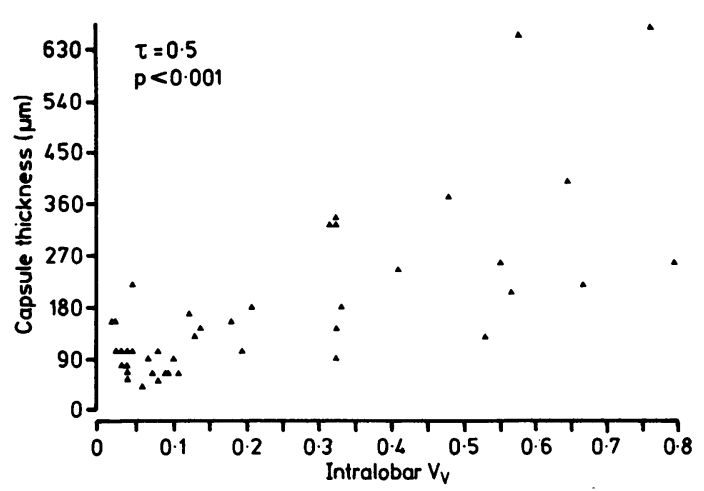

Fig 4 Scatter plot of the volume fraction $\left(V_{V}\right)$ of intralobar non-parenchyma compared with capsular thickness for each of the 42 cases studied.

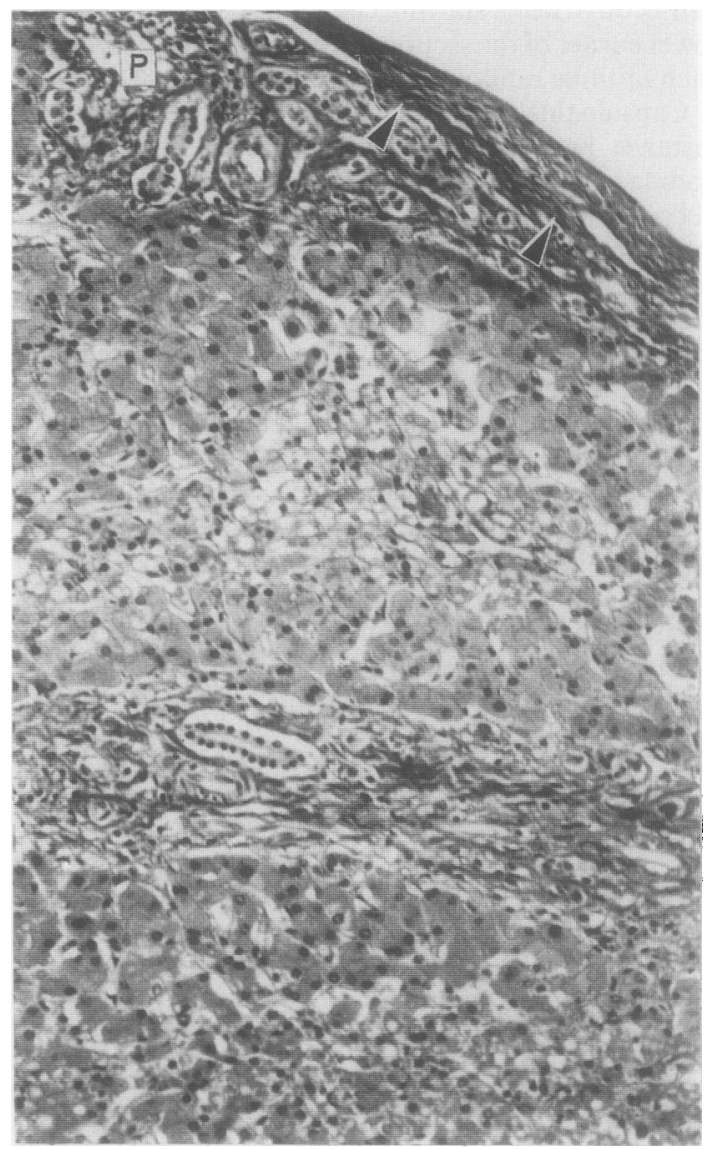

Fig 5 Subcapsular area of fibrotic liver showing similar proliferation of portal tract elements $(P)$ to those in deeper portal areas, and outermost layer (arrowheads) of dense collagenous fibres (see figs 2 and 6). ("Liver trichrome stain".) 


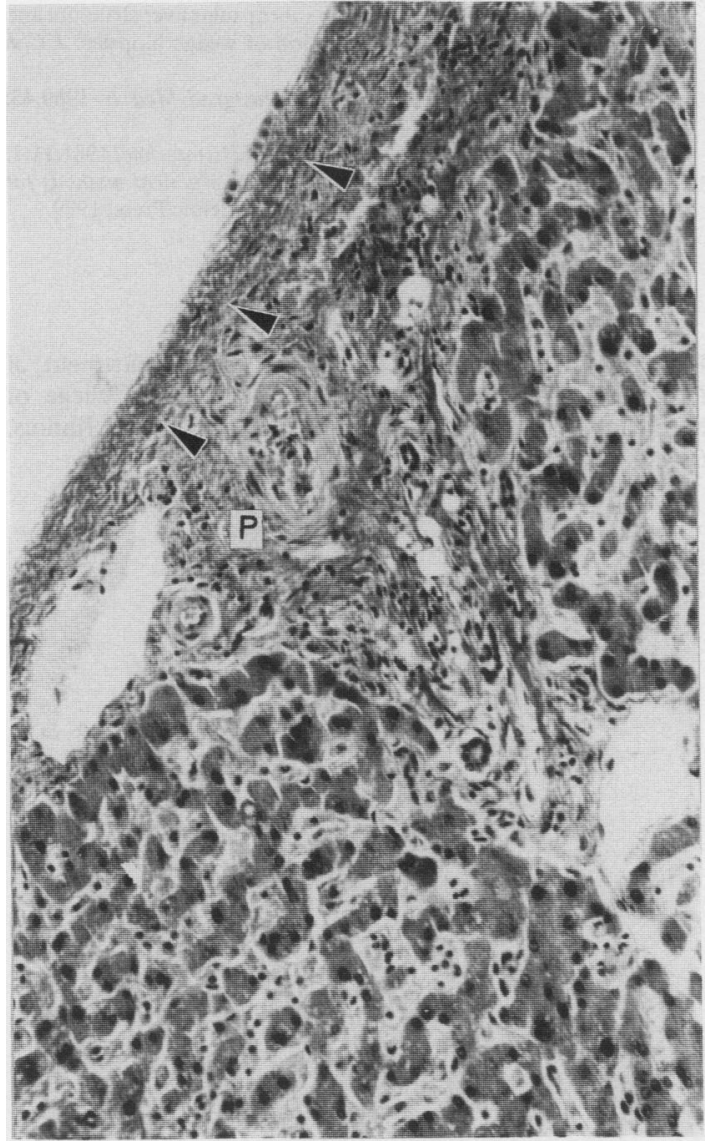

Fig 6 Subcapsular area of normal liver showing existence of a portal tract $(P)$ comprising part of the capsule, and outermost layer (arrowheads) of dense collagenous fibres accompanied by a few cells. ("Liver trichrome stain".)

due to a proliferation of portal tract elementsnamely fibrosis, bile ductules, capillaries, and inflammation (fig 5)-much like that found in the intralobular portal tracts of the same case. An outer layer of dense collagenous fibres, accompanied by a few cells, which was evident in all cases, showed little, if any, thickening (fig 5). In the more or less normal cases the existence of portal tracts extending up to and comprising part of the capsule was occasionally seen (fig 6).

\section{Discussion}

Our results quantitatively show that the amount of subcapsular non-parenchyma correlates directly with the amount of intralobar non-parenchyma. This finding confirms earlier histological studies, ${ }^{5-7}$ and allows us to postulate that the subcapsular non-parenchyma is representative of the rest of the liver. We have also shown that in man there is a significant correlation between capsule thickness and the amount of intralobar non-parenchyma, a correlation that was first shown in our studies on animals. ${ }^{12}$ Our observation that capsule thickening is mainly attributable to the proliferation of portal tract components conforms to a picture of normal liver anatomy, ${ }^{3}$ which shows that there is ramification and continuity of capsular stroma adjacent to hepatic veins and portal tracts.

These data justify the use of subcapsular samples of liver. Even the occasional impression of nodulation arising from small areas of parenchyma, which are immediately beneath the capsule and walled off by fibrosis, can be easily excluded as cirrhotic nodules when the overall subcapsular samples (which can be less than $1 \mathrm{~cm}$ deep) are examined. Given that occasional intralobar nodules can be found adjacent to major portal tracts, we are convinced that both quantitatively and qualitatively there are no essential differences between the subcapsular and intralobar areas of the liver in man.

We considered the possible effect that peritonitis may have had on capsule thickness. Among our cases there were five (four with and one without cirrhosis) that had confirmed peritonitis. Retrospective examination of the data showed that the capsule thicknesses in those livers were no greater than in livers with comparable intralobar fibrosis.

For the purpose of measuring the thickness of the liver capsule, we defined it as the non-parenchymatous area between the liver surface and the hepatocellular parenchyma. There was invariably a layer of dense, collagenous connective tissue and, at times, there was also a layer just below composed of portal tract elements. The thickness of this layer changed in relation to the amount of intralobar non-parenchyma.

Although some may argue that the "true" capsule is represented only by the invariable, outer connective tissue layer, we consider both layers to comprise the capsule, not only by definition in this study, but because of the embryological and anatomical continuity between Glisson's capsule and the portal tracts. ${ }^{3}$ Our data conform to the concept that the amount of diffuse portal expansion like that seen in pre-cirrhotic conditions may be estimated from the amount of capsular thickening. With the rapid and substantial improvements in non-invasive imaging techniques like ultrasonography, a technique for assessing liver non-parenchyma by detecting capsule thickness may eventually be accepted.

We thank Nisha Shah for her technical assistance.

This study was supported in part by the Medical Research Service of the Veterans Administration. 


\section{References}

1 Ryoo JW, Buschmann RJ. A morphometric analysis of the hypertrophy of experimental liver cirrhosis. Virchows Arch (Pathol Anat) 1983;400:173-86.

2 Buschmann RJ, Ryoo JW. Hepatic structural correlates of liver fibrosis: a morphometric analysis. Exp Mol Pathol 1989;50: 114-24.

3 Rappaport AM. Physioanatomic considerations. In: Schiff L, Schiff ER, eds. Diseases of the liver. 6th ed. Philadelphia: J B Lippincott, 1987:1-46.

4 Scheuer PJ. Liver biopsy interpretation. 3rd ed. London: Baillière Tindall, 1980.

5 Ruebner BH, Montgomery CK. Pathology of the liver and biliary tract. New York: John Wiley and Sons, 1982.

6 Ruebner BH. Collagen formation and cirrhosis. Semin Liver Dis 1986;6:212-20.
7 Petrelli M, Scheuer PJ. Variation in subcapsular liver structure and its significance in the interpretation of wedge biopsies. J Clin Pathol 1967;20:743-8.

8 Ansell ID. The uses of liver biopsy. Postgrad Med J 1969;45: 202-7.

9 Dotson W. Liver trichome stain. Tech Bull Histotechnol 1981;11:1.

10 Weibel ER. Stereological methods. Vol. 1: practical methods for biological morphometry. New York: Academic Press, 1979.

Requests for reprints to: Dr J W Ryoo, Department of Pathology, University of Illinois at Chicago, College of Medicine, 1853 West Polk Street, Box 6998, Chicago, Illinois, 60680, USA. 